\title{
Electrostatic discharge in Martian dust storms
}

\author{
Olga Melnik and Michel Parrot \\ Laboratoire de Physique et Chimie de l'Environnement, Centre National de la Recherche Scientifique \\ Orléans, France
}

\begin{abstract}
Although the Martian atmosphere does not satisfy general requirements for lightning generation, there is a possibility of electrical discharge in the case of strong surface winds and a resulting extremely large dust mass loading in the course of large dust storms occurring on this planet. On Earth, negative potential gradients of many thousands of volts per meter have been measured during dust storms when winds are sufficiently strong. However, owing to a lower pressure in the Martian atmosphere, the required voltage for electrical breakdown is lower than on Earth. After a brief review of the observations concerning Earth's atmosphere and the laboratory experiments performed to understand these phenomena, the results of numerical simulation of the electrification in Martian dust storms are presented. Known characteristics of Martian dust grains and the Martian atmosphere are considered, and the different forces applied to the dust particles are taken into account. The electrostatic potential and the evolution of the charge density are calculated using Poisson's equation and the continuity equation. Two different wind configurations in the Martian atmosphere are studied: a wind vortex and a wind parallel to the ground encountering an obstacle (a small hill). It is shown that electric discharge occurs under specific conditions.
\end{abstract}

\section{Introduction}

Electrostatic discharge in planetary atmospheres is an important phenomenon for the evolution of life [Chameides et al., 1979]. On Mars, this phenomenon is very specific because the Martian atmosphere is tenuous, preventing lightning generation, and electrical discharge may only occur in the large dust and sand storms which exist on this planet.

Airborne dust, a significant component of the Martian atmosphere, is a major absorber of solar radiation. The heating of dust affects temperature and pressure and thus the winds. The question arises as to whether the movement of dust during Martian storms constitutes a source of electrification of dust clouds. If this is the case, the following questions arise: What is the mechanism of charge separation? Is there a relationship between particle size and sign of charge? Are potential gradients bigh enough for breakdown? Eden [1977] suggested that Mars has an atmosphere in which clear possible charging mechanisms exist, whereas Rinnert [1995] claimed that it is highly unlikely that efficient electrification and charge accumulation to high potentials occur.

The aim of this paper is to present the results of mathematical modeling of dust electrification during large storms in the low Martian atmosphere. Section 2 gives a brief review of dust and sand storm electrification in terrestrial and Martian atmospheres and considers the peculiarities of contact electrification. The particle size distribution and particle density in the Martian atmosphere are presented in section 3. In section 4, the motion of dust particles is analyzed using Newton's equation, and the charge density is evaluated. The results of the mathematical computer simulations of dust particle movement by wind are given in section 5 , and the conclusions of this work are presented in section 6 .

Copyright 1998 by the American Geophysical Union.

Paper number 98JA01954.

0148-0227/98/98JA-01954\$09.00

\section{Dust and Sand Storm Electrification}

\subsection{Earth}

Electrification in dust clouds during storms is a known phenomenon in the low terrestrial atmosphere. Although the exact cloud charging mechanism is not yet known, data show that dust storms are electrically active and produce remarkable electrical perturbations in the fair-weather electrical state of the atmosphere. In his summary of dust and sand storm electrification, Stow [1969] noted that charged particles and aerosols can be separated by gravity within the column of a dust devil and form an electric dipole. The field observed during strong storms was generally opposite to the average fair-weather field and was as much as $20 \mathrm{kV} \mathrm{m}^{-1} 1 \mathrm{~m}$ above ground. The fair-weather electric field intensity on Earth at ground level is of the order of $100 \mathrm{Vm}^{-1}$ [Uman, 1987]. The electric field recorded during a large dust devil over the Sahara by Freier [1960] indicates that a negative charge density of about $10^{12} \mathrm{el} \mathrm{m}^{-3}\left(1.610^{-7}\right.$

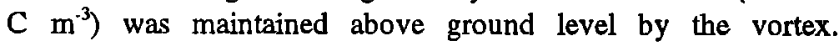
Atmospheric electric potential, space charge, size distribution of dust particles, and other respective parameters were measured by Kamra $[1972,1977]$ in the southwestern United States and in India. Space charge values as high as $10^{11}-10^{12} \mathrm{el} \mathrm{m}^{-3}\left(1.610^{-6}-1.610^{-7} \mathrm{C} \mathrm{m}^{-3}\right)$ of either polarity have been measured $1.25 \mathrm{~m}$ above ground in the United States. During dust storms, both positive and negative potential gradients have been observed at ground level, and both gradients can be associated with the same or opposite sign of space charge. Potential gradients up to $5 \mathrm{kV} \mathrm{m}{ }^{-1}$ have been recorded. During an intensive study in New Mexico, Crozier [1970] measured the electric parameters of 17 dust devils. According to estimates of charge and charge density for eight of these, the negative space charge densities in these dust devils range from $610^{11}$ to $910^{12} \mathrm{el} \mathrm{m}^{-3}$ $\left(-10^{-7}-1.510^{-6} \mathrm{C} \mathrm{m}^{-3}\right)$. The wind velocity at the ground was between 5 and $11 \mathrm{~m} \mathrm{~s}^{-1}$. Space-charge density at high wind velocities was also measured by Israelsson [1994] during the passage of a dust cloud. The weather was sunny and very warm, and the average wind velocity at $10 \mathrm{~m}$ was 3-6 $\mathrm{m} \mathrm{s}^{-1}$. He observed negative space charge 


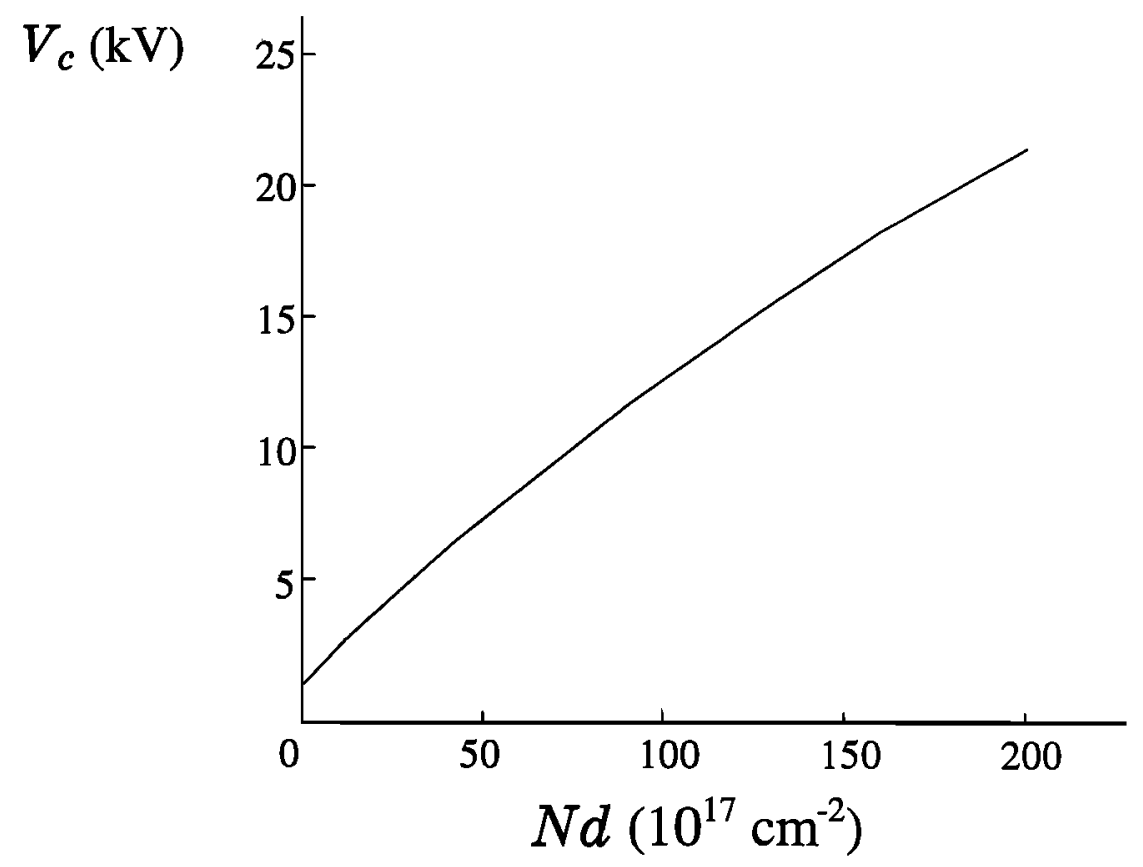

Figure 1. Breakdown voltage as a function of $N d$ for carbon dioxide densities $N$ equal to (1.65 -231$) 10^{17} \mathrm{~cm}^{-3}$ [from Conti and Williams, 1975].

pulses with a duration of about 5 min and a charge density of up to $200 \mathrm{pC} \mathrm{m}^{-3}$, which increased to $300 \mathrm{pC} \mathrm{m}^{-3}$ for a wind speed of 8-9 $\mathrm{m} \mathrm{s}^{-1}$.

\subsection{Mars}

An electrostatic discharge in gases can occur when the potential difference is equal to a given breakdown voltage, but the characteristic parameters of the Martian atmosphere are quite different from those on Earth. The Martian atmosphere is mainly composed of carbon dioxide $\mathrm{CO}_{2}(>95 \%)$, surface pressure is lower than on Earth, of the order of 5.6 mbars $(560 \mathrm{~Pa})$, and temperature is between 140 and $300 \mathrm{~K}$ [Kieffer et al., 1992; Grard, 1995]. The potential difference required for breakdown in gases decreases with gas pressure and depends on the product $N_{a} d$, where $N_{a}$ is gas density and $d$ is the distance between two charges of opposite sign, Paschen's law. Conti and Williams [1975] measured the breakdown potential as a function of $N_{d} d$ for different $\mathrm{CO}_{2}$ densities; their results are shown in Figure 1 for $N_{a}=(1.65-231) 10^{17} \mathrm{~cm}^{-3}$. For an atmospheric density $N_{a} \sim 210^{17} \mathrm{~cm}^{-3}$, potential gradients of only $20-25 \mathrm{kV} \mathrm{m}^{-1}$ are required for breakdown in the Martian atmosphere. On Earth at sea level in a dry atmosphere, this value is $310^{3} \mathrm{kV} \mathrm{m}^{-1}$, whereas in clouds it can be less than $510^{2} \mathrm{kV} \mathrm{m}^{-1}$ [Levin et al., 1983].

Previous work on dust electrification in the Martian atmosphere only concerns laboratory experiments. Eden and Vonnegut [1973] performed an experiment to investigate whether dust becomes electrified when agitated in a $\mathrm{CO}_{2}$ atmosphere at low pressure (10 mbars) and whether any breakdown could be observed as a consequence. They showed visible breakdown and measured potential gradients of $5 \mathrm{kV} \mathrm{m}^{-1}$. This may be applicable to the low Martian atmosphere and shows that electrification of agitated dust can cause visible breakdown in a $\mathrm{CO}_{2}$ atmosphere at low pressure. Eden and Vonnegut [1973] have electrified blown dusts to breakdown voltages with estimated charges of up to $10^{4}$ el per particle, that is 1.6 fC $\left(1 \mathrm{fC}=10^{-15} \mathrm{C}\right)$.

A similar experiment was conducted by Mills [1977], who tested the effect of agitating dry powders at various reduced pressures using a manually rotated 5-L flask. Discharge visible in a darkened room was excited, and he suggested that Martian particles are exposed many times to the cleaning action of this glow discharge and that this process could have been operating discontinuously for a long time.

\subsection{Contact Electrification}

Contact electrification arises when two materials are brought into contact through touching or rubbing; on separation, they are found to be charged [Harper, 1967]. The efficiency of the charge exchange depends on the characteristics of the materials, such as electrical conductivity, permittivity, microstructure of the surface, chemical structure, etc. [Reiter, 1992]. Although contact electrification has been known since classical times, it is still poorly understood. The characteristics of electrification in ice and dust clouds have been observed and measured for a long time, and several laboratory investigations have been conducted to improve the understanding of these phenomena. Different suggestions encountered in the scientific literature concern the relationship between particle size and the sign of obtained charge.

An extensive laboratory study of the charge and size distribution of particles was made by Kunkel [1950], who found that in most cases, positive and negative dust particles were present in equal numbers with only a small amount of neutral particles. The average charge was found to be roughly proportional to the particle radius. The observed charges ranged from 50 to 2500 electrons $(0.4 \mathrm{fC})$ for a particle radius distribution from 0.5 to $30 \mu \mathrm{m}$. According to Kunkel [1950], no correlation exists between size and sign of charge. However, a laboratory experiment by Stow [1969] showed that under normal temperature and humidity conditions, the larger particles were positively charged and the smaller particles were negatively charged. Ette [1971] concluded the same for silica dust clouds, with the larger and heavier particles being positively charged and the smaller and lighter ones being charged negatively. Gravity separates the heavier particles from the lighter ones to produce a dust cloud of negative polarity. Jayaratne [1991] showed that sand grains $(300 \pm 50 \mu \mathrm{m})$ rebounding off an ice surface (impact velocity of $1 \mathrm{~m} \mathrm{~s}^{-1}$ ) remove a 
negative charge, leaving the ice electrically charged. The target charge after collision of a sand grain depends on humidity, temperature, and its velocity, and it was from $0.5 \mathrm{fC}$ near saturation to about $3 \mathrm{fC}$ in a dry environment.

\section{Dust Particles in the Low Martian Atmosphere}

Particle composition, size, and shape determine the optical properties of dust, and based on these visible optical properties measured by spacecraft, silicate is likely to be the main particle constituent. Two attempts were made to obtain dust composition; Toon et al. [1977] said that dust is a mixture of basalt and clay minerals with at least $60 \%$ of $\mathrm{SiO}_{2}$, whereas Hunt [1979] favored a composition of $75 \%$ standard basalt sample and $25 \%$ montmorillonite.

Compared to Earth's atmosphere, two parameters can force particles to move in the Martian atmosphere: (1) reduced atmospheric pressure which enhances the friction velocity and (2) lower gravity. It is thus important to establish the characteristics of the particles which may become suspended in the atmosphere under Martian conditions because suspensible particles will be largely removed from dunes. Edgett and Christensen [1991] determined that an optimum particle diameter of $115 \mu \mathrm{m}$ for the lowest friction velocity threshold $\left(2 \mathrm{~m} \mathrm{~s}^{-1}\right)$ was needed to initiate particle motion for a sand density of $\rho_{p}=2.65$ $\mathrm{g} \mathrm{cm}^{-3}$. This result is in agreement with Hess [1973] and is also discussed by Greeley et al. [1992]. Pollack et al. [1976] located the boundary between saltating and suspensible particles at a particle diameter of about $100 \mu \mathrm{m}$. The estimations were made for particles of $10-$ to $10^{4}-\mu \mathrm{m}$ diameter. On Mars, the radius of the most easily moved particle is about $100 \mu \mathrm{m}$ in diameter, and particles ranging from $<1 \mu \mathrm{m}$ to about $100 \mu \mathrm{m}$ can be put into suspension, while particles between about $100 \mu \mathrm{m}$ and $1 \mathrm{~mm}$ undergo saltation. The thermal structure of the Martian atmosphere during the dissipation phase of the 1971 dust storm was examined by Conrath [1975]. His model considered both gravitational settling and vertical mixing of dust particles and was used to estimate particle size and effective vertical diffusivity. He showed that in the Martian atmosphere a free-falling particle with a radius of 5 - $50 \mu \mathrm{m}$ takes several hours to 10 days to fall to the -5 mbars pressure surface. At higher altitudes, the recent results of the Phobos mission give particle radii between 0.2 and $10 \mu \mathrm{m}$ [Chassefière et al., 1992; Moroz et al., 1993; Murphy et al., 1993].

Levin et al. [1983] measured a particle density close to $5 \mathrm{~cm}^{-3}$ in dust clouds during a severe storm, which is in agreement with Martin [1995]. Chassefiere et al. [1995] obtained similar values when they presented vertical profiles of the most probable distribution of dust radii and density.

The particle size distribution function proposed by Pollack et al. [1979] was obtained from a modified gamma function:

$$
n(r)=c r^{\alpha} \exp \left[-(\alpha / \gamma)\left(r / r_{m}\right)^{\alpha}\right]
$$

where $\mathrm{c}$ is a constant of proportionality and $\alpha$ and $\gamma$ are adjustable parameters: $\alpha=2$ and $\gamma=0.5$. Toon et al. [1977] employ the modal radius $r_{m}$ as a parameter to fit the slope of dust distribution in the range of radii $1-10 \mu \mathrm{m}$ and derived $r_{m}=0.4 \mu \mathrm{m}$, corresponding to effective radius $r_{\text {eff }}=2.75 \mu \mathrm{m}$ and to effective variance $v_{\text {eff }}=0.42$. Inasmuch as this distribution is only applicable for small particles, the classical Gaussian distribution function with a mean value of $50 \mu \mathrm{m}$ can be used for near-surface dust during dust storms, when wind velocities are sufficiently strong.

Direct estimates of wind velocity are extremely rare for Mars. Traveling planetary waves, noted by Zurek et al. [1992], are responsible for short-period fluctuations in average daily surface temperatures and pressures (surface atmospheric pressure varies between $~ 1$ mbar and - 10 mbars depending on topography). They are associated with relatively strong and highly variable winds. Wind velocity near the Martian surface may be as much as $230 \mathrm{~m} \mathrm{~s}^{-1}$ although, as observed by Viking Lander 1 [Kahn et al., 1992], winds of only $30 \mathrm{~m} \mathrm{~s}^{-1}$ (at $1.6 \mathrm{~m}$ above ground) are required to generate sufficient shear stress to lift dust into the atmosphere. The same value was obtained by Leovy et al. [1973] when analyzing global Martian storms in the equatorial region. They observed that the dust was distributed up to $50 \mathrm{~km}$ and that vortex-type winds dominated the 1971 storms. Iversen et al. [1976] suggested that sand particles begin to move when friction velocity, or surface stress, reaches a threshold value, which for Mars is between 1.5 and $2.5 \mathrm{~m} \mathrm{~s}^{-1}$.

In conclusion, it seems very likely that under wind action of -40 $\mathrm{m} \mathrm{s}^{-1}$, particles of density $N=510^{6} \mathrm{~m}^{-3}$ and of radius close to $50 \mu \mathrm{m}$ take part in frictional electrification in the very low Martian atmosphere. These hypotheses used in sections 4 and 5 seem to disagree with previous observations in terms of dust mass loading; although they are possible from theoretical studies. The dust mass loading $M_{d}$ is the dust mass in a vertical column that extends throughout the atmosphere and that has a horizontal cross section of unity. It can be expressed as $N m H$, where $m$ is the mass of a particle with average radius and $H$ is the height of the column. A rough calculation with our hypotheses gives $M_{d} \sim 150,000 \mathrm{~kg} \mathrm{~km}^{-2}$, whereas the maximum value observed on Mars by Martin [1995] during local dust storms is $-18,000 \mathrm{~kg} \mathrm{~km}^{-2}$. However, in section 5 we will consider only the effect of dust devils very close to the Martian surface $(-100 \mathrm{~m})$, and we will suppose that particles of radius close to $50 \mu \mathrm{m}$ are lifted in the atmosphere up to $\sim 100 \mathrm{~m}$ altitude. The charge of each sand grain could be $-1 \mathrm{fC} \mu \mathrm{m}^{-1}$ of radius.

\section{Numerical Simulation}

\subsection{Dust Particle Motion During Dust Storms}

To determine whether electrostatic discharge takes place in the low Martian atmosphere during dust storms, it is necessary to know the number of collisions between dust particles moving under wind action and then to calculate the electric field induced by the charge density to check if the breakdown voltage could be reached.

The dust particles are considered as spherical with a radius $r_{p}$, mass $m_{p}$, velocity $\mathbf{v}_{\mathrm{p}}$, and charge $q_{p}$. Particle movement is given by Newton's equation:

$$
m_{p} \frac{d \mathbf{v}_{\mathbf{p}}}{d t}=\mathbf{F}_{\mathbf{F}}+\mathbf{F}_{\mathbf{G}}+\mathbf{F}_{\mathbf{d}}
$$

where $\mathbf{F}_{\mathbf{F}}$ is the force due to the electromagnetic field:

$$
\mathbf{F}_{\mathbf{F}}=q_{p} \mathbf{E}
$$

in this equation the term $v_{p} \times B$ is neglected because the magnetic field $B$ is very weak. $F_{G}$ is the force of gravity:

$$
\mathbf{F}_{\mathbf{G}}=m_{p} \mathbf{g}
$$

where $g$ is the constant of gravity for Mars and $g=3.72 \mathrm{~m} \mathrm{~s}^{-2} . F_{d}$ is the drag force due to the viscosity [Edgett and Christensen, 1991]:

$$
\mathbf{F}_{\mathrm{d}}=\frac{1}{2} C_{d} \pi r_{p}^{2} \rho_{a} \mathbf{v}_{\mathbf{i}}^{2}
$$

where $C_{d}$ is the drag coefficient which is related to the Reynolds number and $v_{i}$ is the relative velocity $\left(v_{i}=v_{w}-v_{p}\right.$, where $v_{w}$ is the wind velocity). The atmospheric density $\rho_{a}=1.610^{-2} \mathrm{~kg} \mathrm{~m}^{-3}$ [Santee and 


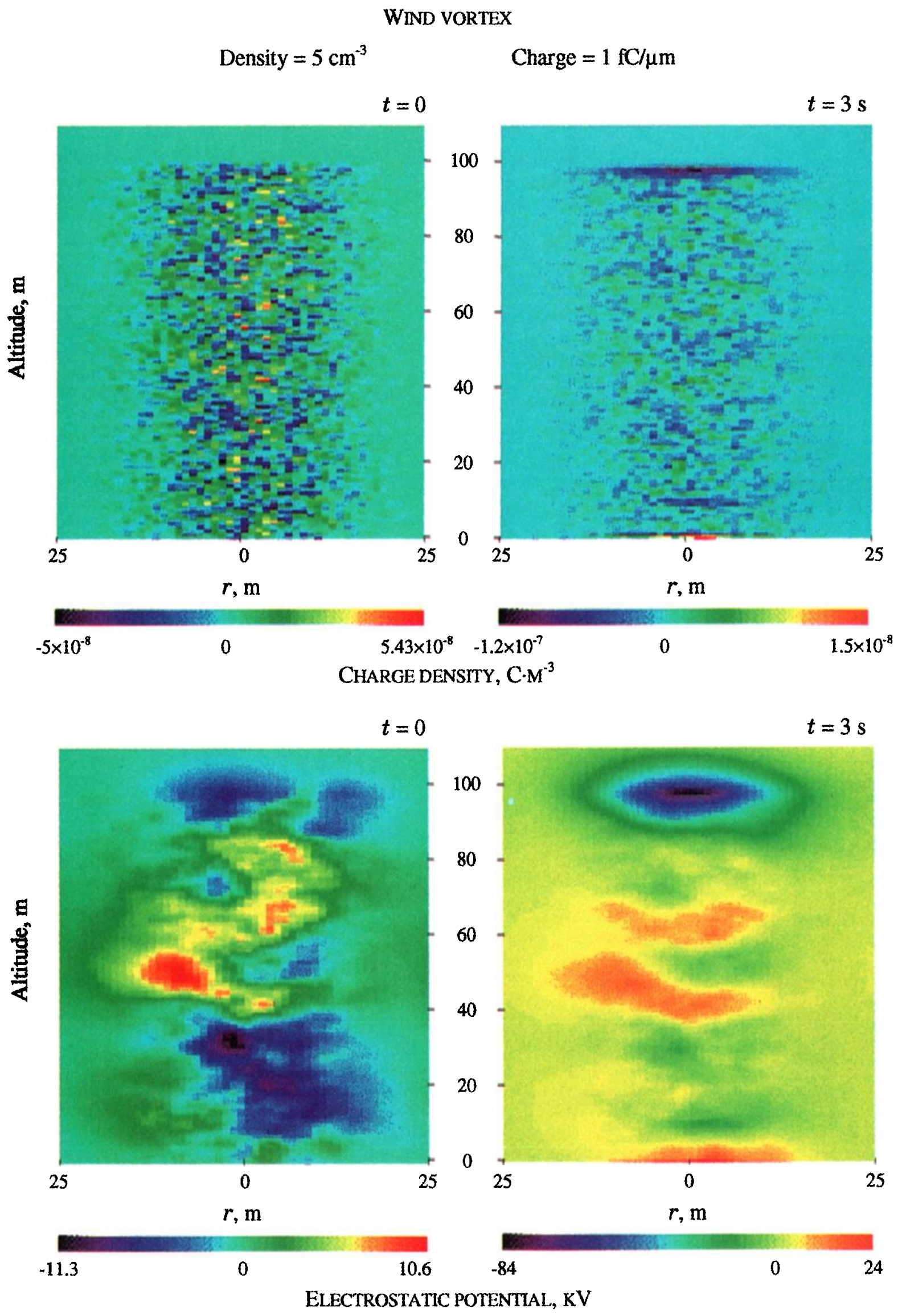

Plate 1. Charge density and electrostatic potential at different times during the simulation. 


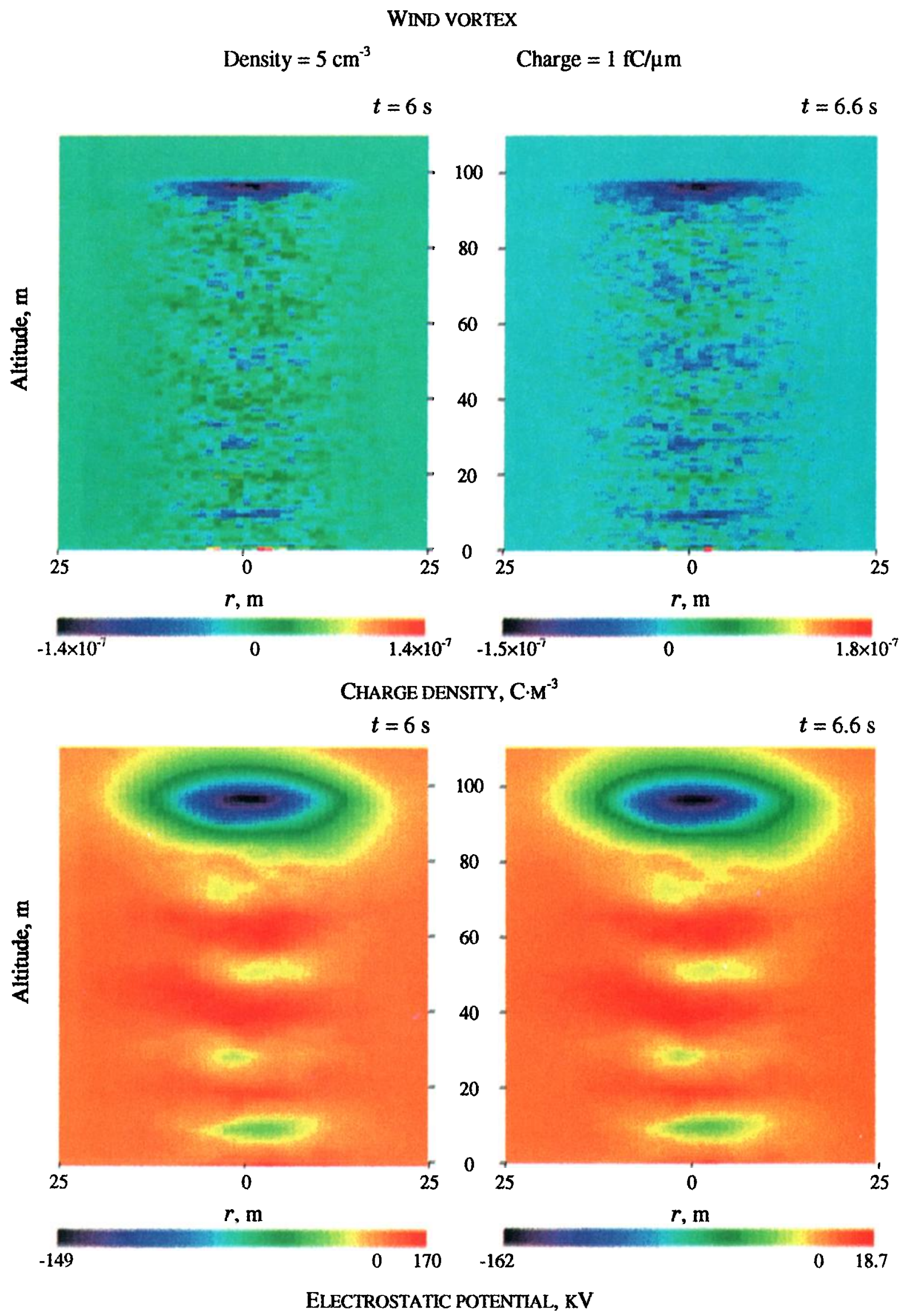

Plate 1. (continued) 

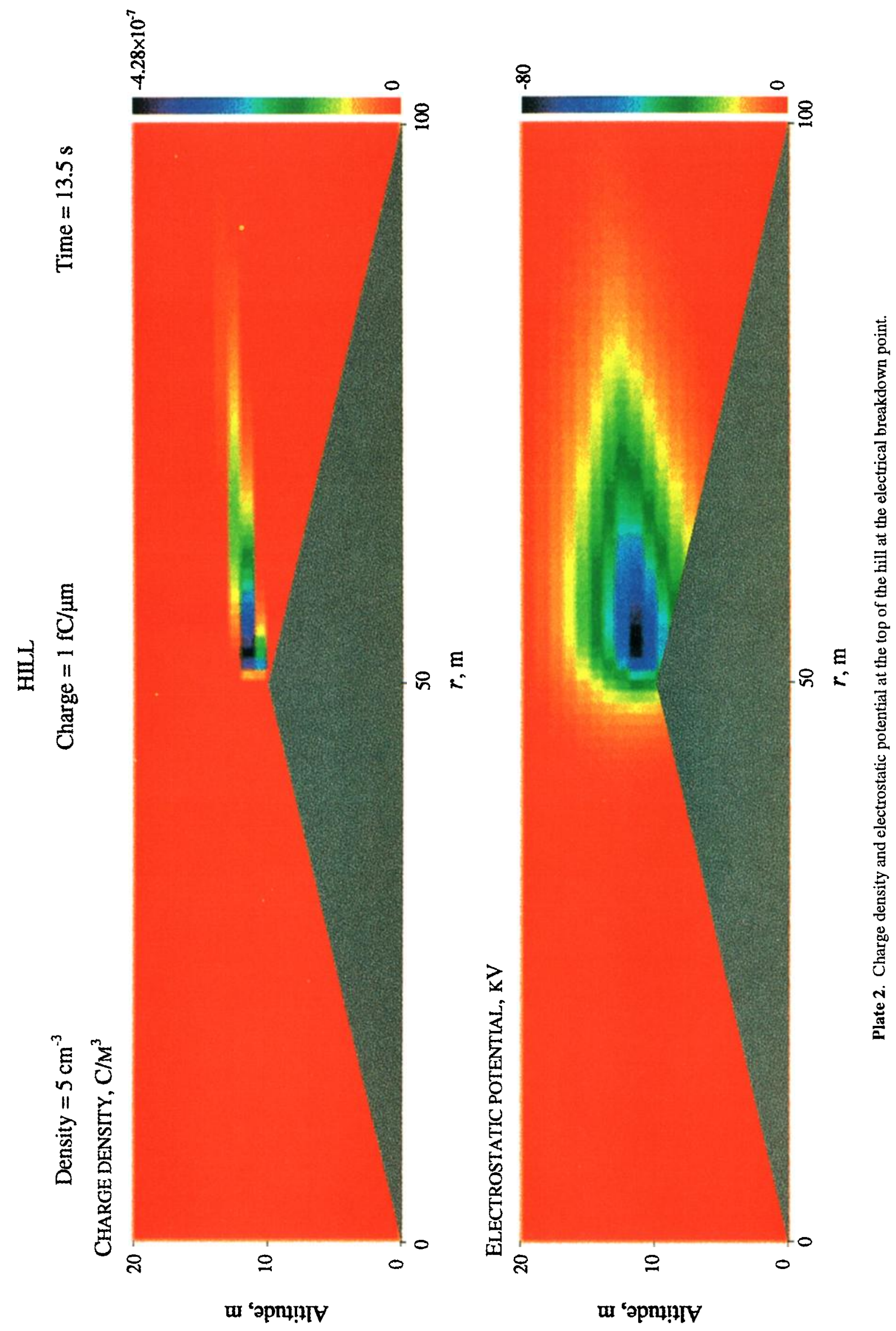
Crisp, 1995]. For high wind velocity, we assume that $C_{d} / 2$ is $\sim 1$, and $F_{d}$ is written as $F_{w}$ the force due to the wind:

$$
\mathbf{F}_{w}=\left(\mathbf{v}_{w}-\mathbf{v}_{p}\right)^{2} \pi r_{p}^{2} \rho_{a}
$$

For very low wind velocity, and therefore small Reynolds number, the drag force given by (5) can be written

$$
\mathbf{F}_{\mathbf{d}}=6 \pi \eta r_{p} \mathbf{v}_{\mathbf{p}}
$$

where $\eta$ is the Martian atmospheric viscosity $\left(\eta-1.510^{-5} \mathrm{k} \mathrm{m}^{-1} \mathrm{~s}^{-1}\right)$ [Santee and Crisp, 1995]. When the wind force decreases, the sedimentation process then comes into play. Aerodynamic drag tends toward keeping the particle aloft, while the gravity tends to pull it downward. When the process is fixed, the left term of (2) is equal to zero, and the equation $F_{d}=F_{G}$, where $F_{d}$ is given by (7) and $F_{G}$ is given by (4), provides the velocity of sedimentation:

$$
\mathbf{v}_{\mathrm{s}}=\frac{2 r_{p}^{2} g \rho_{p}}{9 \eta}
$$

where $\rho_{p}$ is the particle density $\left(\rho_{p}=2.6510^{3} \mathrm{~kg} \mathrm{~m}^{-3}\right)$.

\subsection{Electrification Mechanism}

The space-time variation of the quasi-electrostatic field is given by

$$
\mathbf{E}=-\nabla \varphi
$$

where $\varphi$ is the electrostatic potential. This potential $\varphi$ and the charge density $\rho$ are related by the continuity equation:

$$
\frac{\partial \rho}{\partial t}-\nabla[\sigma \nabla \varphi]=0
$$

and Poisson's equation:

$$
\nabla^{2} \varphi=-\left(\rho+\rho_{s}\right) / \varepsilon_{0}
$$

where $\rho_{s}(\mathbf{r}, t)$ is the source charge density due to the charge of the new dust particles arriving in the system, $\sigma$ is the magnitude of the atmospheric electrical conductivity in the vicinity of the Martian surface, and $\epsilon_{0}$ is the vacuum permittivity. This approach is similar to that of Pasko et al. [1995, 1996], who studied the electric field distribution in thunderstorm clouds in order to understand the electrostatic discharge observed in the upper atmosphere.

\section{Results}

Two wind configurations are considered: a vortex and a wind parallel to the Martian surface encountering a small hill. A first simulation was done to check that the particles collide under wind action and to determine the number of collisions that occur in a wind vortex as a function of particle density and wind velocity. Equation (2) was used but taking only gravity force given by (4) and wind force given by (6) into account. The simulation box measured $10 \mathrm{~cm}$ $\times 10 \mathrm{~cm} \times 10 \mathrm{~cm}$, and the number of particles inside was $10^{3}, 510^{3}$, and $10^{4}$, representing a particle density $N$ of 1,5 , and $10 \mathrm{~cm}^{-3}$, respectively. The results, that is the number of collisions per cubic meter and per second, are shown in Table 1 for three different wind velocities.

As expected, the number of collisions increases with wind velocity and particle density. In this first simulation, only particle movement
Table 1. Number of Collisions per Cubic Meter and per Second in a Wind Vortex.

\begin{tabular}{cccc}
\hline & \multicolumn{3}{c}{$N, \mathrm{~m}^{-3}$} \\
\cline { 2 - 4 } $\mathrm{v}_{\mathrm{w}}, \mathrm{m}_{\mathrm{s}} \mathrm{s}^{-1}$ & $10^{6}$ & $5 \times 10^{6}$ & $10^{7}$ \\
\hline 30 & $5.75 \times 10^{4}$ & $1.12 \times 10^{5}$ & $7.77 \times 10^{6}$ \\
40 & $8.68 \times 10^{4}$ & $2.12 \times 10^{6}$ & $8.8 \times 10^{6}$ \\
60 & $9.2 \times 10^{4}$ & $2.77 \times 10^{6}$ & $1.13 \times 10^{7}$ \\
\hline
\end{tabular}

under wind action and gravity is considered so as to study the collisions and to assign a charge to each particle. When two particles collided, the lighter one acquired a negative charge proportional to its radius $\left(1 \mathrm{fC} \mathrm{m}^{-1}\right)$, and the heavier one acquired the same charge but with the opposite sign.

In the second simulation, at time $t=0$, we assume that the vortex is established and that, based on past wind action, both light and heavy dust particles are distributed in the volume. The selected dust particle density is $510^{6} \mathrm{~m}^{-3}$, and the average wind velocity $40 \mathrm{~m} \mathrm{~s}^{-1}$. The particles have radii given by a Gaussian distribution centered on 50 $\mu \mathrm{m}$. All particles are then charged according to the first simulation; the sum of the charges is zero. In the following simulation, at time $t$ $=0$, the wind stops and the particles are under the action of forces given by (3), (4), and (7). We assume a closed simulation system (no dust particles introduced from above). The details concerning the numerical simulation to solve (2), (9), (10), and (11) are given in the appendix. Equation (2) is considered with $\mathbf{F}_{\mathrm{d}}$ given by (7). Plate 1 presents the charge density and electrostatic potential results of this simulation as a function of time for the vortex configuration; the intensities are color-coded according to the scale at the bottom of each plot. At $t=0$, the charges are randomly distributed up to $100 \mathrm{~m}$, and no particular shape is recognized for the electrostatic potential. At $t=3 \mathrm{~s}$, the heavier particles, which are generally positively charged, have already fallen from the upper levels, and a negative charge appears at the top of our simulation grid. The potential varies and increases accordingly. At $t=6 \mathrm{~s}$, particle movement still continues under the action of gravity and the increasing electric field. The zone of maximum charge density decreases in altitude, and layers of positive and negative charge start to emerge below. This is more evident at $t=6.6 \mathrm{~s}$, which represents the end of the simulation because the breakdown of the electric field $\left(20 \mathrm{kV} \mathrm{m}^{-1}\right)$ is reached. With this layered distribution, we can expect other discharges before all the dust particles reach the surface.

Figure 2 represents the evolution of the maximum of the electric field during the simulation. It should be noted that the position of this maximum moved in our simulation grid as a function of time (which explains the inflexion point in the curve). The electric field quickly increases at the beginning, evolves up to the breakdown of the electric field, and then becomes smoother. The electric field is relatively high $\left(-3 \mathrm{kV} \mathrm{m}^{-1}\right)$ at the beginning, which is due to the random distribution of the charges in the cells of our simulation grid. This is a known phenomenon in numerical plasma simulation [Dawson, 1983]. We tried to achieve a "quiet start" by reordering the grains at $t=0$ randomly in the cells in pairs. The two particles are located at the same place in the cell, and have charges of equal absolute value but with opposite signs (but not necessarily the same radius). The results are shown in Figure 3 where the maximum of the electric field is plotted as a function of time. The electric field is null at the beginning, but at $t=0.1 \mathrm{~s}$, the electric field is much lower ( $400 \mathrm{~V} \mathrm{~m}^{-1}$ ) than in Figure 2. The breakdown was reached after a similar time $(7.2 \mathrm{~s})$ to the previous simulation with this different start. 


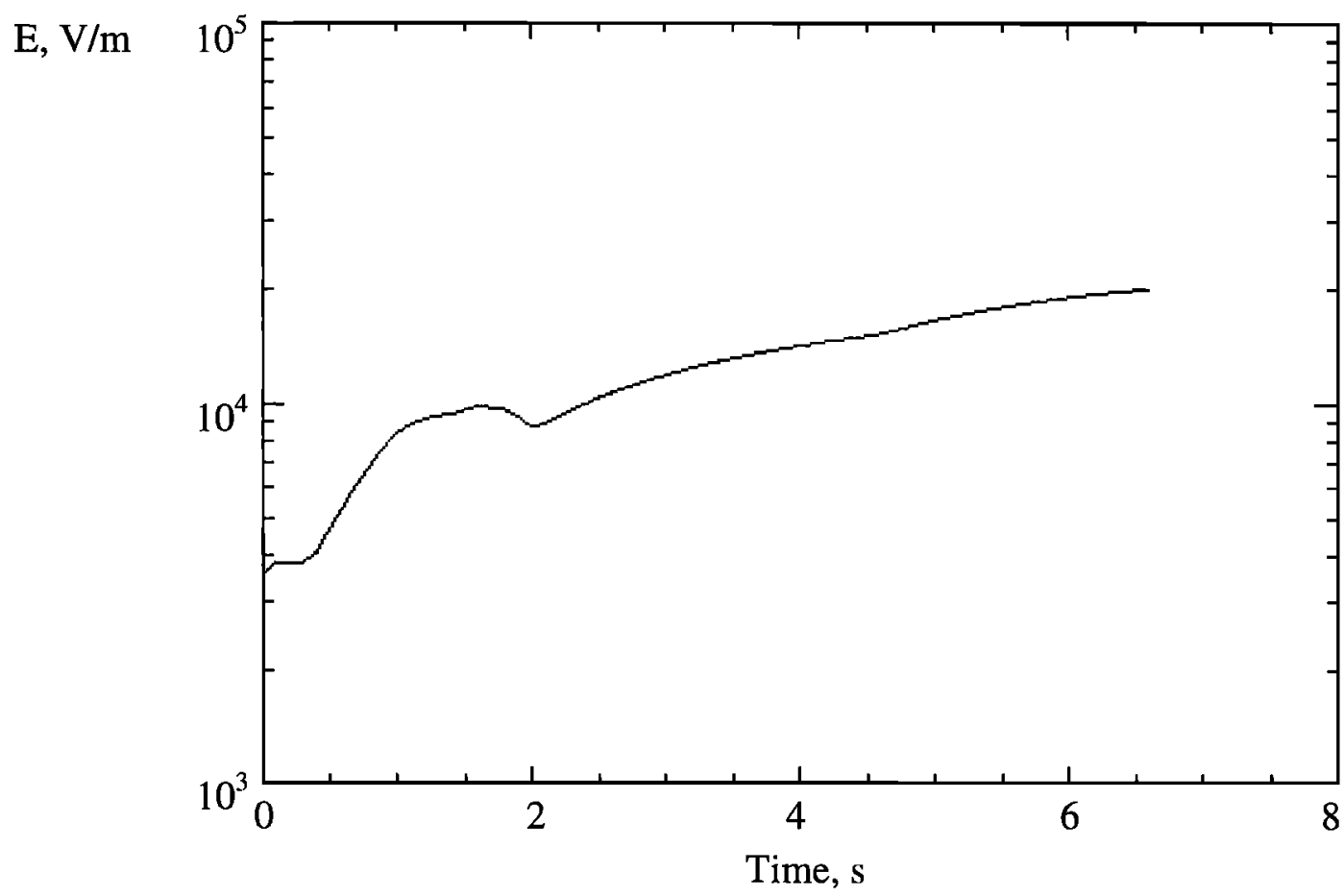

Figure 2. Evolution of the maximum of the electric field in the simwation grid as a function of time.

Two other simulations were then carried out with different parameters: a first one with a reduced density $N=2 \mathrm{~cm}^{-3}$ and a grain

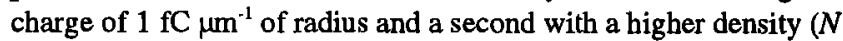
$\left.=5 \mathrm{~cm}^{-3}\right)$ and a reduced grain charge $\left(0.5 \mathrm{fC} \mu \mathrm{m}^{-1}\right.$ of radius $)$. Electrical breakdown was reached in both cases but only after a considerable increase in time, $t=72 \mathrm{~s}$ and $t=97 \mathrm{~s}$, respectively.

For the second type of wind configuration, the wind is parallel to the Martian surface and encounters a small hill. The wind follows the slope topography and carries away the sand grains from the foot of the hill. Wind velocity is $40 \mathrm{~m} \mathrm{~s}^{-1}$. The grains roll up the hill because of the wind action and become negatively charged by friction (1 $\mathrm{fC}$ $\mu \mathrm{m}^{-1}$ of radius). We suppose that the dust grains are spherical and that the slope is easy $\left(11^{\circ}\right)$ and smooth. The first part of the simulation concerns particle movement on the hill in order to determine the radius of the particles that reach the top. This also made it possible to determine the velocity of the particles at the top of the hill and the time taken to climb it. We assume that the only forces involved are wind and gravity. Further details are given in the appendix. When the

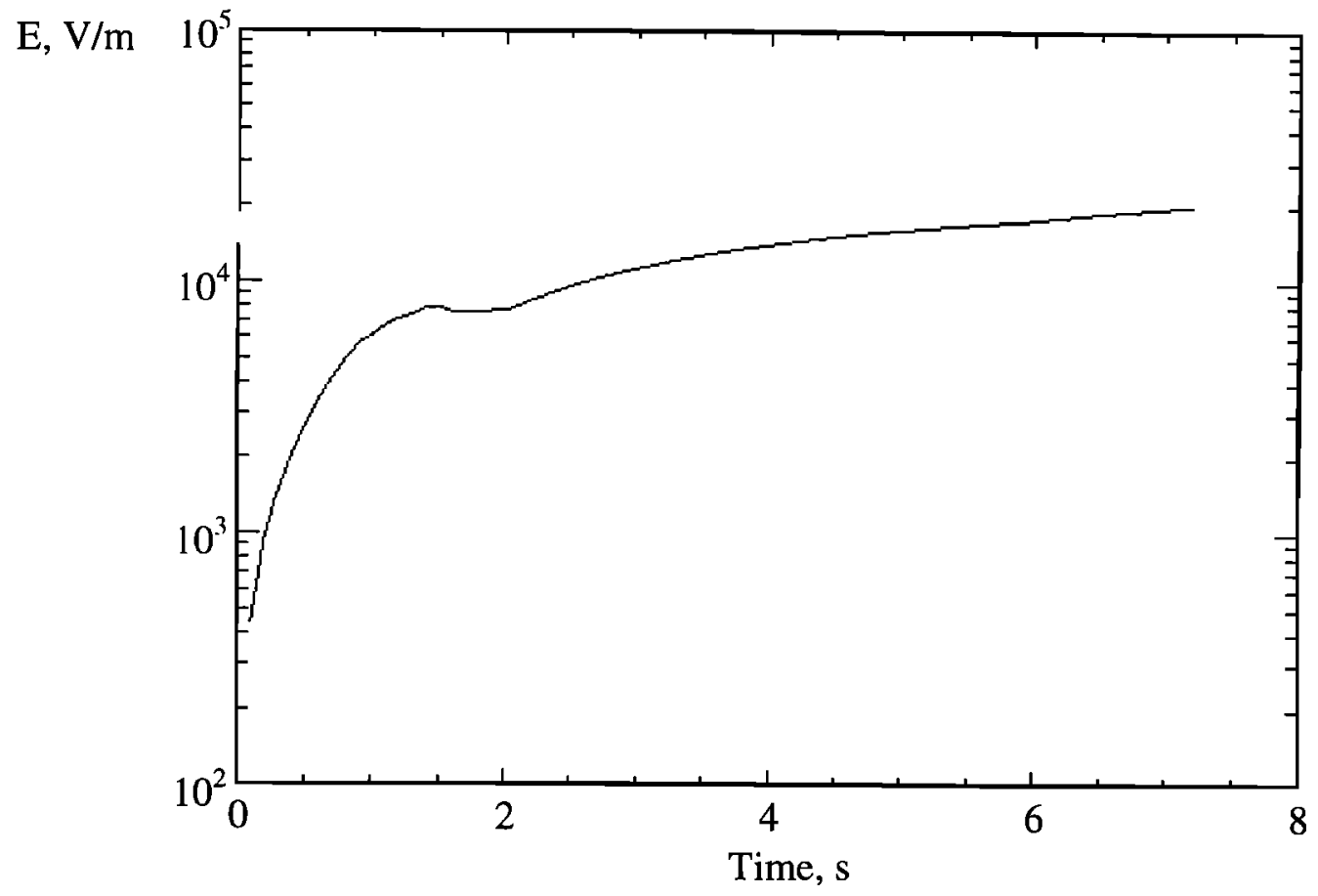

Figure 3. Evolution of the maximum of the electric field in the simulation grid as a function of time with a "quiet start". 


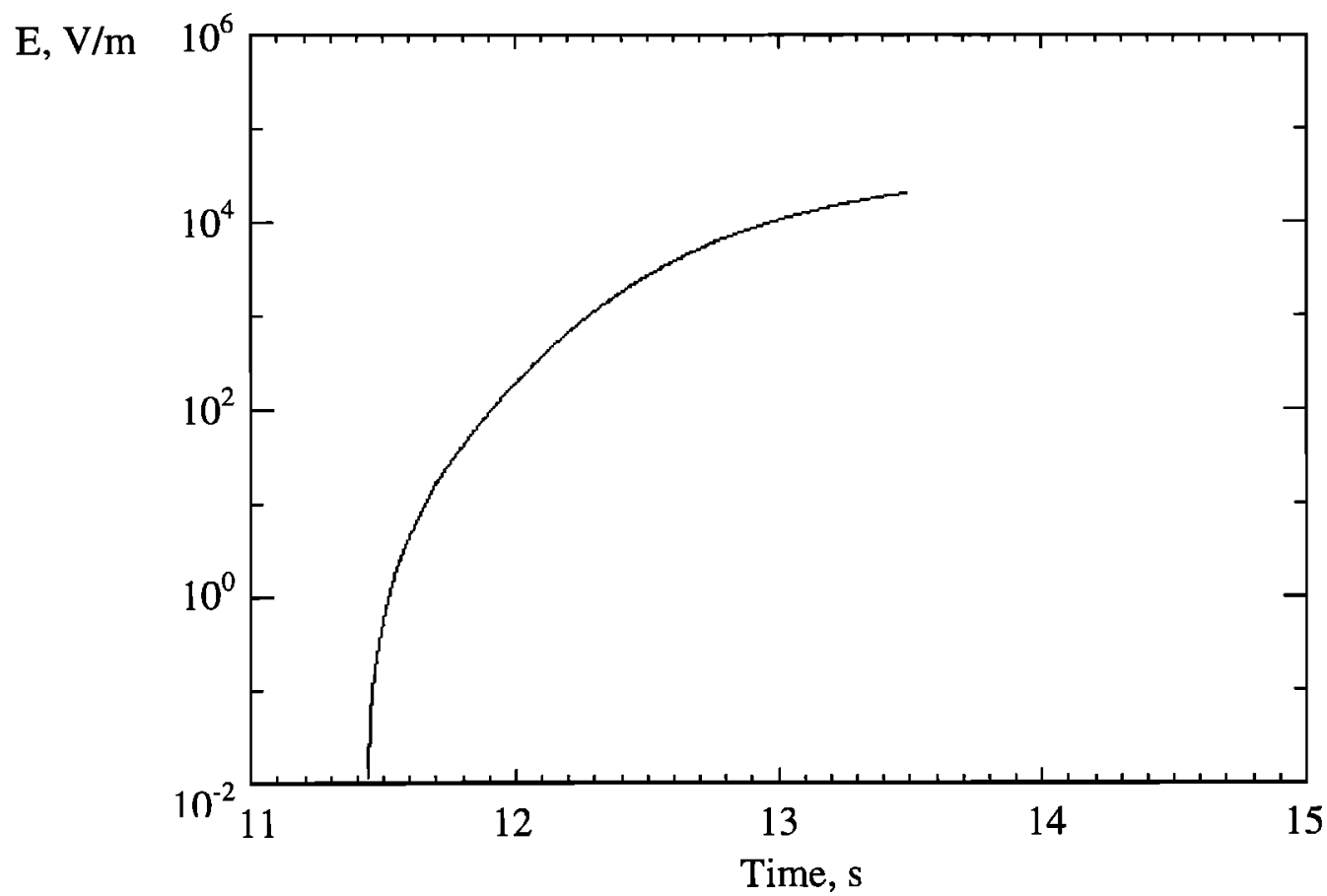

Figure 4. Evolution of the maximum of the electric field at the top of the hill as a function of time.

particles reach the top they are carried away by the wind, and as they are charged, they contribute to the formation of a charge density. Particle density at the top is $N=5 \mathrm{~cm}^{-3}$. The results of charge density and electrostatic potential at the point of electrical breakdown are given in Plate 2; the intensities are color-coded according to the scale on the right. Time $t=0$ corresponds to the time when the first particle starts to climb the hill. The hill is represented in grey in Plate 2. Breakdown is seen to occur close to the top of the hill when the particles start to fly away in the wind. Figure 4 shows the evolution of the maximum of the electric field in the simulation grid. Growth is exponential and very regular, and breakdown is quickly reached. However, the results of another simulation not shown here with a reduced wind velocity $\left(30 \mathrm{~m} \mathrm{~s}^{-1}\right)$ indicate that breakdown is reached after a much longer time and that many particles fall onto the other side of the hill because of gravity.

\section{Conclusions}

On the basis of the known characteristics of Martian dust and past laboratory experiments, we have studied the charge that dust particles can acquire through collisions ( $1 \mathrm{fC} \mu \mathrm{m}^{-1}$ of radius) under wind action. It is shown that the breakdown electric field is lower in the Martian atmosphere than on Earth. Two different wind configurations are considered: a vortex which carries dust particles into the atmosphere and wind parallel to the surface encountering a hill causing particles to roll up the slope and be ejected at the top. In both cases, the simulation results indicate that it is possible to reach breakdown in the Martian atmosphere under certain conditions of particle density and wind velocity. However, the time to reach breakdown is much longer with a decrease in particle density, individual charge of a grain, or wind velocity. This means that other processes not considered in the simulation may occur, such as a recombination of charges through subsequent collisions. Therefore breakdown will not be attained.

Electrical discharge is able to generate electromagnetic waves propagating in the atmosphere and then in the ionosphere.
Observation of these waves by electric antennae on a lander would not be too difficult if the dust storm is not too far from the lander, but observation with an orbiter will depend on ionospheric parameters and wave frequencies. The propagation of electromagnetic waves in the Martian ionosphere assuming a source of radiation in the atmosphere is the subject of a forthcoming paper. Concerning visual spectrometry, a camera on a low-altitude orbiter $(300 \mathrm{~km})$ would certainly see these discharges on the nightside.

This work was undertaken as part of the wave experiment Electromagnitnoe Issledovanie Marsa (ELISMA) on board the MARS-96 mission [ELISMA Experimenters, 1998]. The main scientific objective of ELISMA was to study the electromagnetic waves in the Martian ionosphere, but unfortunately, the launch of MARS-96 failed. A new mission is planned for 1998: the Japanese mission PLANET-B with a camera and another wave experiment [Tsuruda et al., 1996].

\section{Appendix: Numerical Calculations of the Electric Field}

Section A1 and A2 present the numerical solutions of (2), (9), (10), and (11) for the two wind configurations: a vortex and a horizontal wind encountering an obstacle.

\section{A1. Vortex}

The computational grid is characterized by its length $X(50 \mathrm{~m})$ and its height $Z(100 \mathrm{~m})$. This grid is split into cells of selected dimensions $\Delta x$ and $\Delta z(1 \mathrm{~m})$. The number of particles in our simulation is $N_{p}=10^{6}$. At the beginning, the dust particles are uniformly distributed in the $z$ direction (the number of particles in each level is identical), and their positions relative to the $x$ axis are given by a Gaussian distribution centered at $X / 2$, the central axis of the vortex. Knowing the number of particles and their charge in the cell defined by the grid points $(i, j),(i+1, j),(i+1, j+1)$, and $(i, j+1), i t$ 
is possible to obtain the contribution of each particle to the source charge densities $\rho_{S}(\mathbf{i}, \mathbf{j}), \rho_{s}(\mathbf{i}+1, \mathrm{j}), \rho_{s}(\mathbf{i}+1, \mathbf{j}+1)$, and $\rho_{S}(\mathbf{i}, \mathbf{j}+1)$ by summation with a weighting factor [Birdsall and Langdon, 1991]. To take into account the dimensions of our simulation system, the charge density is corrected with the factor $N X Z / N_{p}$ to give an effective charge per unit length.

Poisson's equation (11) is solved using the fast Fourier transform (FFT) method [Birdsall and Langdon, 1991] because it is more convenient to work in $k$ space. The two-dimensional Fourier transforming of the total charge density $\rho_{h,}$ gives $\rho_{h m}$, and using the same system, the potential can easily be calculated with

$$
\varphi_{h m}=\frac{\rho_{l, m}}{\epsilon_{0} K_{l, m}^{2}}
$$

where

$$
K_{l, m}^{2}=k_{x}^{2} \sin c^{2}\left(\frac{k_{x} \Delta x}{2}\right)+k_{z}^{2} \operatorname{sinc}{ }^{2}\left(\frac{k_{z} \Delta z}{2}\right)
$$

and $\operatorname{sinc}(x)=\sin (x) / x$. The coefficients $k_{x}$ and $k_{z}$ are equal to $2 \pi l / X$ and $2 \pi \mathrm{m} / Z$, respectively. Then the inverse FFT takes back $\varphi_{b m}$ in the real space $\varphi_{i j}$. The electric field is obtained using equation (9) with a fivepoint method [Birdsall and Langdon, 1991]:

$$
\mathrm{E}_{\mathrm{i}, \mathrm{j}}=\left[\left\{\frac{\varphi_{i-1, j}-\varphi_{i+1, j}}{2 \Delta x}\right\}^{2}+\left\{\frac{\varphi_{i, j-1}-\varphi_{i, j+1}}{2 \Delta z}\right\}^{2}\right]^{1 / 2}
$$

The continuity equation (10) gives the variation of the charge density

$$
\mathrm{d} \rho_{i, j}=\sigma\left\{\frac{\varphi_{i-1, j}-2 \varphi_{i, j}+\varphi_{i+1, j}}{\Delta x^{2}}+\frac{\varphi_{i, j-1}-2 \varphi_{i, j}+\varphi_{i, j+1}}{\Delta z^{2}}\right\}_{\mathrm{d} t}
$$

where $\sigma=10^{-11} \mathrm{~S} \mathrm{~m}^{-1}$ [Grard, 1995], and $\mathrm{d} t$ is the time step of the simulation $(0.1 \mathrm{~s})$. We now need to calculate the new positions of the particles in each level when submitted to constraints. We assume that the forces are mainly directed along the $z$ axis. In the absence of wind, the main forces are drag and electrical. Using (2), the particle velocity can be written at time $t$

$$
\mathbf{v}_{\mathrm{p}, \mathrm{t}}=\frac{q_{p} \mathrm{E}_{\mathrm{t}}}{m_{p}} \mathrm{~d} t+\mathbf{v}_{\mathrm{s}}
$$

where $\mathrm{p}$ is the particle indice and $\mathrm{v}_{\mathrm{s}}$ is the sedimentation velocity given by (8). The intensity of the electric field is weighted as before to take into account the position of the particles inside the cell. The new vertical position of each particle is then given by

$$
z_{p, t+d t}=\mathrm{v}_{\mathrm{p}, t} \mathrm{~d} t+z_{p, t}
$$

With (17) we can calculate the number of particles in each level taking into consideration the loss of particles that fall and the gain from higher levels and evaluate the new charge density due to the dust particles. At time $t+\mathrm{d} t$, the new total density charge is given by

$$
\rho_{t+d t}=\rho_{t}+\mathrm{d} \rho
$$

where $\mathrm{d} \rho$ is given by (15). The system of equations (equations (12), (14), (16), (17), and (18)) is repeated in order to check if the breakdown voltage is reached. The process is then stopped because the total charge density dramatically changes after a discharge.

\section{A2. Horizontal Wind Encountering a Hill}

The computation grid is characterized by its length $X(100 \mathrm{~m})$ and its height $Z(20 \mathrm{~m})$. The hill is $10 \mathrm{~m}$ high and $100 \mathrm{~m}$ long, with a slope of $\sim 11^{\circ}$. The particle velocity has two components along $x$ and $z$, and can be expressed as

$$
\mathbf{v}_{\mathrm{p}, \mathrm{t}+\mathrm{d} \mathrm{t}}=\left(\frac{q_{p} \mathbf{E}_{\mathrm{t}}}{m_{p}}+\frac{\mathbf{F}_{\mathbf{w}}}{m_{p}}+\mathrm{g}\right) \mathrm{d} t+\mathbf{v}_{\mathrm{p}, \mathrm{t}}
$$

where $F_{w}$ is given by (6). The resolution of the equations used is similar to that in section $A 1$, except for the change of the charge density at $t+\mathrm{d} t$ because new particles are continuously introduced into the system at the top of the hill. This is written as

$$
\rho_{t+d t}=\rho_{S_{t+d t}}+\rho_{t}+\mathrm{d} \rho
$$

where $\rho_{\mathrm{St}+\mathrm{dl}}$ represents the change due to the new charges and $\mathrm{d} \rho$ is given by (15).

Acknowledgment. The Editor thanks Michael Flasar and Eric Chassefiere for their assistance in evaluating this paper.

\section{References}

Birdsall, C.K., and A.B. Langdon, Plasma Physics via Computer Simulation, Adam Hilger, Bristol, England 1991.

Chameides, W.L., J.C.G. Walker, and A.F. Nagy, Possible chemical impact of planetary lightning in the atmospheres of Venus and Mars, Nature, 280, 820-822, 1979.

Chassefière, E., J.E. Blamont, V.A. Krasnopolsky, O.I. Korablev, S.K. Atreya, and R.A. West, Vertical structure and size distributions of Martian aerosols from solar occultation measurements, Icarus, 97, 46-69, 1992.

Chassefière, E., P. Drossart, and O.I. Korablev, Post-Phobos model for the altitude and size distribution of dust in the low Martian atmosphere, J. Geophys. Res., 100, 5525-5539, 1995.

Conrath, BJ., Thermal structure of the Martian atmosphere during the dissipation of the dust storm of 1971, Icarus, 24, 36-46, 1975.

Conti, VJ., and A.W. Williams, Ionization growth in carbon dioxide, J. Phys. D Appl. Phys., 8, 2198-2207, 1975.

Crozier, W.D., Dust devil properties, J. Geophys. Res., 75, 4583-4585, 1970.

Dawson, J.M., Particle simulation of plasmas, Rev. Modern Phys., 55, 403-447, 1983.

Eden, H.F., Review of electrification processes in planetary atmospheres, in Electrical Processes in Atmosphere, edited by $\mathbf{H}$. Dolezalek and R. Reiter, pp. 567-573, Steinkopff, Darmstadt, Germany, 1977.

Eden, H.F., and B. Vonnegut, Electrical breakdown caused by dust motion in low-pressure atmospheres: Considerations for Mars, Science, 180, 962-963, 1973.

Edgett, K.S. and P.R. Christensen, The particle size of Martian aeolian dunes, J. Geophys. Res., 96, 22,765-22,776, 1991.

ELISMA Experimenters, The wave complex on the MARS-96 orbiter: ELISMA, Planet. Space Sci., 46, 701-713, 1998.

Ette, A.I.I., The effect of the Harmattan dust on atmospheric electric parameters, J. Atmos. Terr. Phys., 33, 295-300, 1971.

Freier, G.D., The electric field of a large dust devil, J. Geophys. Res., $65,3504,1960$.

Grard, R., Solar photon interaction with the Martian surface and related electrical and chemical phenomena, Icarus, 114, 130-138, 1995.

Greeley, R., N. Lancaster, S. Lee, and P. Thomas, Martian aeolian processes, sediments, and features, in Mars, edited by H.H. Kieffer et al., pp. 730-766, Univ. of Ariz. Press, Tucson, 1992.

Harper, W.R., Contact and Frictional Electrification, Clarendon Press, Oxford, England, 1967. 
Hess, S. L., Martian winds and dust clouds, Planet. Space Sci., 21, 1549-1557, 1973.

Hunt, G.E., Thermal infrared properties of the Martian atmosphere, J. Geophys. Res., 84, 2865-2874, 1979.

Israelsson, S., Measurements of surface-air space charges carried by dry wind-driven dust, J. Atmos.Terr. Phys., 56, 1551-1556, 1994.

Iversen, J.D., R. Greeley, and J.B. Pollack, Windblown dust on Earth, Mars, and Venus, J. Atmos. Sci., 33, 2425-2429, 1976.

Jayaratne, E.R., Charge separation during the impact of sand on ice and its relevance to theories of thunderstorm electrification, Atmos. Res., 26, 407-424, 1991.

Kahn R.A., T.Z. Martin, R.W. Zurek, and S.W. Lee, The Martian dust cycle, in Mars, edited by H.H. Kieffer et al., pp. 1017-1054, Univ. of Ariz. Press, Tucson, 1992.

Kamra, A.K., Measurements of the electrical properties of dust storms, J. Geophys. Res., 77, 5856-5869, 1972.

Kamra, A.K., Effect of dust-raising winds on the atmospheric electric field, in Electrical Processes in Atmosphere, edited by $\mathrm{H}$. Dolezalek and R. Reiter, pp. 168-174, Steinkopff, Darmstadt, Germany, 1977.

Kieffer, H.H., B.M. Jakosky., and C.W. Snyder, The planet Mars: From antiquity to the present, in Mars, edited by H.H. Kieffer et al., pp. 1-33, Univ. of Ariz. Press, Tucson, 1992.

Kunkel, W.B., The static electrification of dust particles on dispersion into a cloud, J. Appl. Phys., 21, 820-832, 1950.

Leovy, C. B., R.W. Zurek, and J.B. Pollack, Mechanisms for Mars dust storms, J. Atmos. Sci., 30, 749-762, 1973.

Levin, Z., W. Borucki, and O. Toon, Lightning generation in planetary atmospheres, Icarus, 56, 80-115, 1983.

Martin, T. Z., Mass of dust in the Martian atmosphere, J. Geophys. Res., 100, 7509-7512, 1995.

Mills, A.A., Dust clouds and frictional generation of glow discharges on Mars, Nature, $268,614,1977$.

Moroz, V. I., E.V. Petrova, and L.V. Ksanfomality, Spectrophotometry of Mars in the KRFM experiment of the Phobos mission: Some properties of the particles of atmospheric aerosols and the surface, Planet. Space Sci., 41, 569-585, 1993.

Murphy, J.R., R.M. Haberle, O.B. Toon, and J.B. Pollack, Martian global dust storms: Zonally symmetric numerical simulations including size-dependent particle transport, J. Geophys. Res., 98, 3197-3220, 1993.

Pasko, V.P., U.S. Inan, Y.N. Taranenko, and T.F. Bell, Heating, ionization and upward discharges in the mesosphere due to intense quasi-electrostatic thundercloud fields, Geophys. Res. Lett., 22, 365-368, 1995.

Pasko, V.P., U.S. Inan, and T.F. Bell, Blue jets produced by quasielectrostatic pre-discharge thundercloud fields, Geophys. Res. Lett., 23, 301-304, 1996.

Pollack, J.B., R. Haberle, R. Greeley, and J. Iversen, Estimates of the wind speeds required for particles motion on Mars, Icarus, 29. 395-417, 1976.

Pollack, J.B., D.S. Colbum, F.M. Flasar, R. Kahn, C.E. Carlston, and D. Pidek, Properties and effects of dust particles suspended in the Martian atmosphere, J. Geophys. Res., 84, 2929-2945, 1979.

Reiter, R., Phenomena in atmospheric and environmenal electricity, in Developments in Atmospheric Science, pp. 20-25, Elsevier, New-York, 1992.

Rinnert, K., Lightning within planetary atmospheres, in Handbook of Atmospheric Electrodynamics, vol. 1, edited by Hans Volland, pp. 203-233, CRC Press, Boca Raton, Flo., 1995.

Santee, M.L., and D. Crisp, Diagnostic calculations of the circulation in the Martian atmosphere, J. Geophys. Res., 100, 5465-5484, 1995.

Stow, C.D., Dust and sand storm electrification, Weather, 24, 134 $140,1969$.

Toon, O.B., J.B. Pollack, and C. Sagan, Physical properties of the particles composing the Martian dust storm of 1971-1972, Icarus, 30, 663-696, 1977.

Tsuruda, K., I. Nakatani, and T. Yamamoto, Planet-B mission to MARS-1998, Adv.Space Res., 17 (12), 21-29, 1996.

Uman, M.A., The Lightning Discharge, Int. Geophys. Ser., vol. 39, Academic, San Diego, Calif., 1987.

Zurek, R.W., J.R. Barnes, R.M. Haberle, J.B. Pollack, J.E. Tillman, and C.B. Leovy, Dynamics of the atmosphere of Mars, in Mars, edited by H.H. Kieffer et al., pp. 835-933, Univ. of Ariz. Press, Tucson, 1992.

O. Melnik and M. Parrot, Laboratoire de Physique et Chimie de l'Environnement, CNRS, 3A, Avenue de la Recherche Scientifique, 45071 Orléans cedex 02, France (omelnik@cnrs-orleans.fr, mparrot@cnrs-orleans.fr)

(Received November 4, 1997; revised May 19, 1998;

accepted May 22, 1998.) 